\title{
Unusual presentations of ulnar nerve paresthesia: Mycotic aneurysm and leprosy
}

\author{
Susan D Moffatt BSc MD, Winston S Parkhill MD FRCSC \\ Division of Plastic Surgery, Victoria General Hospital, Dalhousie University, Halifax, \\ Nova Scotia
}

\begin{abstract}
SD Moffatt, WS Parkhill. Unusual presentations of ulnar nerve paresthesia: Mycotic aneurysm and leprosy. Can J Plast Surg 1996;4(4):217-220. Ulnar nerve compression causing clinical symptoms is a common occurrence. There are numerous conditions that can cause compression. Recently two very interesting and unusual etiologies were seen at the Plastic Surgery service. Leprosy causing ulnar nerve compression is a rare occurrence in a Canadian hospital, and so is a case of palmar mycotic aneurysm in the postantibiotic era.
\end{abstract}

Key Words: Leprosy, Mycotic aneurysms, Ulnar nerve

Tableau inhabituel de paresthésie du nerf cubital : anévrisme mycotique et lèpre

RÉSUMÉ : Les symptômes cliniques dûs à une compression du nerf cubital sont courants. De nombreuses maladies peuvent causer une telle compression. Récemment, deux étiologies très intéressantes et très rares ont pu être observées au service de chirurgie plastique. La lèpre provoquant une compression du nerf cubital est un rare phénomène dans un hôpital canadien, tout autant que l'anévrisme mycotique palmaire à notre époque post-antibiotique.

The ulnar nerve is the continuation of the medial cord of the brachial plexus and includes $\mathrm{C} 8$ and $\mathrm{T} 1$ nerve roots. It enters the forearm by passing between the medial epicondyle of the humerus and the olecranon, where it is very superficial and easily traumatized (1). In the distal part of the forearm, the ulnar nerve again becomes relatively superficial. Near the pisiform bone it pierces the deep fascia and passes superficial to the flexor retinaculum where it ends by dividing into the superficial and deep branches in Guyon's canal. It again becomes relatively susceptible to extrinsic compression (2).

Ulnar nerve compression is the second most common compressive lesion of the upper extremity after carpal tunnel syndrome (1). The effect of compression applied to a peripheral nerve varies with the magnitude as well as the duration of the compressive trauma. Depending on the size and location of the fibres within the nerve trunk, nerve fibres show varying susceptibility to compression. Large fibres are more vulnerable to compression and ischemia than small fibres (3).

In 1861, Guyon described the ulnar tunnel at the wrist and concluded that this area may be the site of pathological lesions causing compression syndromes. The 
etiologies of ulnar nerve compression have been found to range from common conditions, such as ganglions, anesthetic palsy and arthritis, to rare conditions, such as osteochondromatosis of the elbow, leprosy, spindle cell carcinoma and ulnar artery disease (2).

Diagnosis of ulnar compression syndrome may be difficult because of patients' complaints of vague elbow pain, odd paresthesias and minimal physical findings. History and clinical examinations are important, and electrodiagnostical studies are often very useful. Surgical exploration, in view of the probable existence of a space occupying lesion in many cases, should be instigated without hesitation (3).

\section{CASE PRESENTATION}

\section{Case 1}

A 41-year-old male presented to the Cardiovascular Surgery Service for emergency mitral valve repair secondary to bacterial endocarditis-induced valve rupture. Preoperative transthoracic echocardiogram had revealed two vegetations on the mitral valve. Blood cultures taken at that time grew alpha hemolytic streptococcus, and the patient was started on a regimen of penicillin $\mathrm{G}$ and gentamicin. Histological findings of the replaced valve were in keeping with bacterial endocarditis. Past medical history was significant for a dental abscess six months before surgery which was treated with extraction and antibiotics. Thereafter he developed night sweats, weight loss and extreme lethargy.

Approximately two weeks after his valve replacement surgery, the patient complained of right palm tenderness and burning. He also complained of swelling and paresthesia along the ulnar aspect of his right hand extending into the fourth and fifth digits. On examination, he was afebrile. Head and neck examination was normal. Cardiopulmonary examination was also normal with the exception of findings secondary to the cardiac surgery. Examination of the right hand revealed a palmar erythematous swelling near the hypothenar eminence (Figure 1). There was a notable decrease in sensation in the ulnar nerve distribution. Motor strength was normal, and a bruit was audible over the swelling. There were no other clinical findings in keeping with additional aneurysms. All hematological indices were normal. Ultrasound revealed a $2.5 \times 2.5 \times 1.8 \mathrm{~cm}$ aneurysm of the ulnar artery in the proximal aspect of the palm.

The Infectious Diseases Service put forth the working diagnosis of a mycotic aneurysm secondary to his recent bacterial endocarditis. Under the care of both Plastic Surgery and Cardiovascular Surgery, the patient was taken to the operating room for exploration. Using a palmar incision which was extended to a carpal tunnel incision, the palmar vascular arch was exposed. A large aneurysm involving the superficial arch was identified and found to extend from the princeps pollicis to the musculature to the hypothenar eminence (Figure 2). The superficial arch of the ulnar artery was identified as it passed through Guyon's canal into the palm, and the superficial arch of the radial artery was identified as it passed through the adductor canal. Both were ligated, and the distal superficial branches passing along the metacarpals were identified and ligated, thus freeing the mycotic aneurysm in its entirety (Figure 3). All nerve structures of the medial and ulnar nerve were preserved. Hemostasis was assured, and a primary closure was performed. 

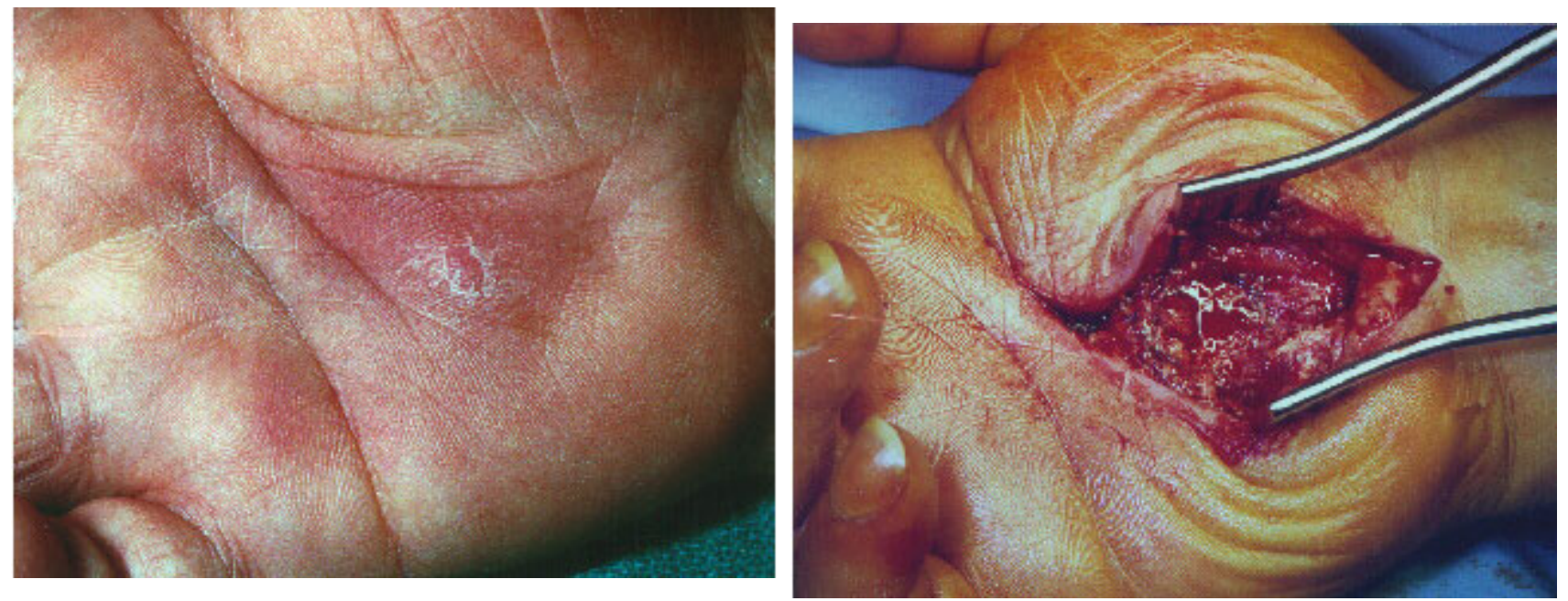

Figure 1) Mycotic aneurysm presenting as a pulsatile mass with an audible bruit
Figure 2) Mycotic aneurysm involving the superficial palmar arch

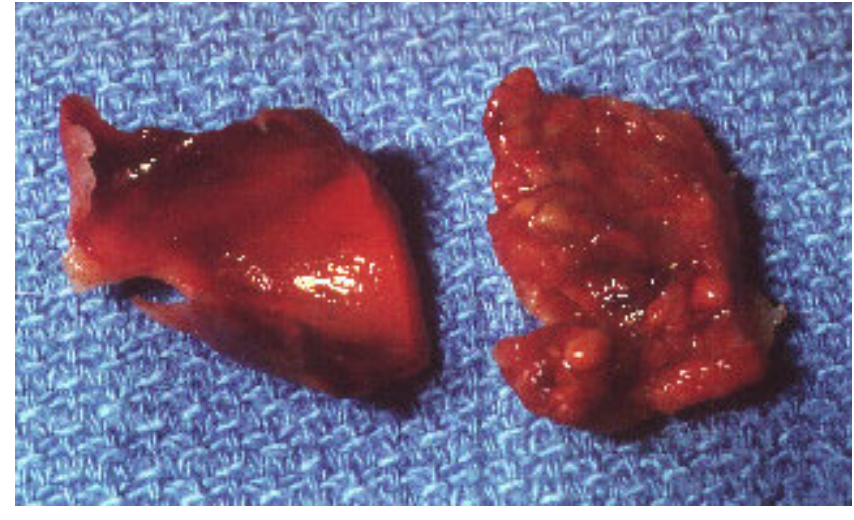

Figure 3) Excised mycotic aneurysm revealing large intraluminal diameter

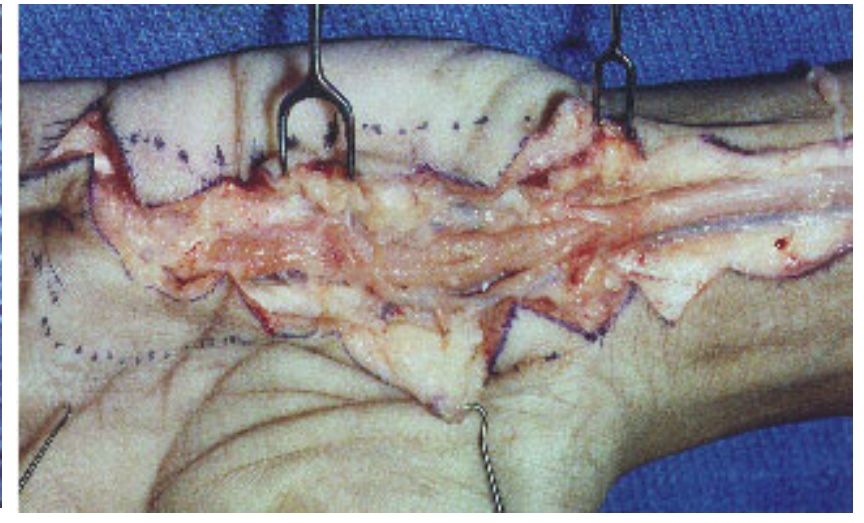

Figure 4) Exposed ulnar nerve infected with Mycobacterium leprae

After the operation, the patient did very well. Cultures of the aneurysm tissue and sequential blood cultures were negative. The patient regained full sensation in the ulnar distribution. The patient completed a six-week regimen of penicillin and gentamicin. At the time of follow-up, the patient did not have any signs or symptoms of recurrent mycotic aneurysms. 


\section{Case 2}

A 41-year-old male of Philippine origin presented to the Plastic Surgery Service with a two-month complaint of numbness in the ulnar aspect of his left palm. This numbness initially involved only the fifth digit and gradually spread to involve the fourth digit and lateral palm. An erythematous, coin-sized lesion was initially present on the palm but had since disappeared. He felt that his fifth digit was weak, but that his overall grip strength was unchanged. He denied fever, chills, night sweats or weight loss. He had no known exposure to tuberculosis or leprosy, but did have a very extensive travel history. Past medical history was significant for a lower gastrointestinal bleed 20 years previous that was conservatively treated.

On physical examination he was afebrile. Head and neck examination were negative for adenopathy or lesions. Cardiopulmonary examination was unremarkable. His right hand was normal. His left hand revealed anesthesia in the ulnar distribution and a palpable linear mass near the hypothenar eminence. There was obvious weakness of the fifth digit. Further examination revealed three small discrete papules along his right flank and one erythematous, scaly lesion on his right lateral leg. All hematological studies were normal. Chest and hand x-rays were normal. Electromyelography nerve conduction studies were abnormal and revealed a conduction block at the elbow with axonal degeneration.

The patient was taken to the operating room for ulnar nerve exploration. The ulnar nerve was first exposed, identified at the elbow and was noted to be quite firm, fibrotic and enlarged. This ulnar nerve was transposed anteriorly. The palmar mass was then explored through a palmar incision, and an enlarged ulnar nerve was identified (Figure 4). The motor branches appeared to be normal but the sensory nerves were grossly abnormal, as were the fourth and fifth digital nerves. Nerve biopsies were taken, and the incision closed primarily.

Subsequent pathology of the nerve biopsies revealed granulomatous lesions highly suggestive of tuberculoid leprosy. All cultures, including skin lesion samples, were negative. Infectious Diseases suggested a regimen of dapsone $100 \mathrm{mg}$ orally once daily and rifampin $600 \mathrm{mg}$ orally once monthly for a minimum of six months. The patient regained reasonable motor activity of the hand, but had residual sensory and motor deficit in the ulnar distribution. The patient returned home where follow-up hopefully continues.

\section{DISCUSSION}

In 1885, Sir William Osler presented his classic report to the Royal College of Physicians in London on "Malignant Endocarditis" (4). He used the term mycotic aneurysm to describe infected aneurysms that had developed as a complication of bacterial endocarditis (5). Today mycotic aneurysm is a term used when referring to endocarditis associated aneurysms, microbial arteritis, infection of already existing aneurysms and post-traumatic infected false aneurysms. In recent years the true incidence of mycotic aneurysms has increased because of increasing numbers of immune suppressed hosts, invasive hemodynamic monitoring, angiography and drug addiction (5). In general the diagnosis of mycotic aneurysm is based on the classical features of fever, pain, positive blood cultures and a pulsatile mass. 
Mycotic aneurysms are seen in 3\% to $15 \%$ of patients with endocarditis and are more commonly seen secondary to hematogenous seeding of arteries during bacteremia or due to inoculation of bacteria at time of local arterial trauma such as arterial catheters $(6,7)$. The infection weakens the arterial wall, and the natural history of extremity mycotic aneurysms is to enlarge, compressing surrounding tissues, and rupture (8). Mycotic aneurysms may occur at multiple sites in any given patient but certain anatomical locations, such as the abdominal and thoracic aorta and intracranial vessels, predominate (6). The main extracranial, extrathoracic sites of predilection are the iliofemoral segments. Involvement of the upper extremity vasculature is indeed rare, with only 10 cases being reported over the past 20 years (8).

Histological examination of mycotic aneurysms reveals a picture of organizing thrombus and necrosis of the vessel wall with varying degrees of destruction of the vascular layers. The bacteriology of mycotic aneurysms is predominantly Staphylococcus aureus and Salmonella species (9). Gram-negative involvement is associated with a greater virulence and a higher incidence of aneurysmal rupture (5). Blood cultures are reported as being positive in $46 \%$ of cases and aneurysmal cultures positive in $73 \%$ of cases (6). Vascular ultrasound is the best diagnostic tool, with angiography used to map the vascular supply before surgery (7).

Treatment of mycotic aneurysms begins with preoperative antibiotics. Aggressive and appropriate antibiotic therapy should be continued four to six weeks. Early surgical intervention is of utmost importance because the risk of rupture for infected vascular segments is as high as $75 \%$ (7). Aneurysms of the upper extremity are usually removed by obtaining proximal and distal control of the involved vessel with ligation and excision of the injured artery. It is further recommended that vascular reconstruction be performed only in patients in whom ischemia develops following excision of the aneurysm $(4,8)$. If reconstructive surgery is required, the use of autologous vein or artery is suggested because the more serious complication of graft failure is encountered mainly in patients who undergo prosthetic graft repair $(6,10)$.

Leprosy or Hansen's disease is a chronic granulomatous infection caused by Mycobacterium leprae which attacks superficial tissues, especially the skin and peripheral nerves $(11,12)$. The introduction of multidrug therapy by the World Health Organization (WHO) in 1982 has resulted in a more than 50\% reduction in the caseload worldwide (13). The epidemiology of the disease is poorly understood, yet it is recognized as a major public health problem in most countries of Africa, Asia and Latin America. Ninety per cent of the cases diagnosed in the United States in the past two decades have occurred in immigrants from leprosy-endemic countries (6). The chief portal of exit is thought to be the nasal mucosa of untreated lepromatous patients with subsequent direct human to human transmission. The incubation period is frequently three to five years, but has also been reported to range from six months to several decades $(11,12)$.

Leprosy consists of a spectrum in which five groups are identified. All groups are based on the Ridley-Jopling immunological classification (11). One pole is that of Tuberculoid Leprosy (TT) consisting of patients with a high level of cell-mediated immunity. At the other pole are patients with Lepromatous Leprosy (LL) who have specific cellular immunodeficiencies to various antigens of $M$ leprae. Most patients, however, are in the Borderline categories and include Borderline Tuberculoid (BT), 
Borderline Lepramatous (BL) and mid-Borderline (BB). Two additional groups are the indeterminate form (I) and a pure neural form. To facilitate WHO multidrug therapy implementation, it has been recommended that two groups be identified. Paucibacillary leprosy refers to initial skin smear-negative cases only and includes TT, BT and I cases. Multibacillary leprosy refers to initial skin smear-positive cases and includes all BB, BL and LL cases $(11,12)$.

Damage caused by leprosy may occur in mucous membranes, eyes, lymph nodes and skin with nerves being invariably damaged (11). The three main functions of nerves (sensory, motor and autonomic) may be equally affected, but usually the sensory component is the earliest and the most severely affected. When paresthesia and anesthesia develop, the hands and feet are prone to trauma which often results in deformity and mutilation. When peripheral nerves are involved they become enlarged, a very rare finding in other clinical conditions. The ulnar nerve is the most commonly affected peripheral nerve, followed by the median and radial and common peroneal nerves $(1,2)$.

Leprosy can mimic many dermatological and neurological disorders, so a high index of suspicion is required to make an accurate diagnosis. The diagnosis is based on the presence of one or more of the cardinal signs: anesthetic skin lesions, enlargement of peripheral nerves and the presence of acid-fast bacilli in smears taken from the skin lesions (12).

Multidrug therapy regimes have been outlines by WHO. In paucibacillary leprosy, monthly rifampin plus daily dapsone is given for six months. For multibacillary leprosy, monthly rifampin and clofazimine together with daily self-administered clofazimine and dapsone are given for a minimum of two years and preferably until the patient achieves smear negativity $(13,14)$.

\section{REFERENCES}

1. Rayen GM. Proximal ulnar nerve compression. Hand Clin 1992;8:325-35.

2. Moneim MS. Ulnar nerve compression at the wrist. Hand Clin 1992;8:337-43.

3. Lundborg G, Dahlin LB. The pathophysiology of nerve compression. Hand Clin 1992;8:215-25.

4. Flamand F, Harris KA, DeRose GM, et al. Arteritis due to salmonella with aneurysm formation: Two cases. Can J Surg 1992;35:248-52.

5. Gomes MN, Choyke PL, Wallace RB. Infected aortic aneurysms.

Ann Surg 1992;215:435-42.

6. Johnson JR, Ledgerwood AM, Lucas CE. Mycotic aneurysm.

Arch Surg 1983;118:577-82.

7. Kearney RA, Eisen HJ, Wolf JE. Nonvalvular infections of the cardiovascular system. Ann Intern Med 1994;121:219-30.

8. Berrettoni BA, Seitz WH. Mycotic aneurysm in a digital artery:

Case report and literature review. J Hand Surg 1990;15A:305-8.

9. Vyas SK, Law NW, Loehry CA. Mycotic aneurysm of left subclavian artery. Br Heart J 1993;69:455-6.

10. Pasic M, Carrel T, Von Segesser L, et al. In situ repair of mycotic aneurysm of the ascending aorta. J Thorac Cardiovasc Surg 1993;105:321-6. 
11. Ji B, Grosset J. Leprosy. In: Hoeprich PD, Jorden MC, Ronald AR, eds. Infectious Diseases, 5th edn. Philadelphia: JB Lippincott Company, 1994:1008-17.

12. Miller RA. Leprosy (Hansen's disease). In: Wilson JD, Braunwald E, Isselbach KJ, et al, eds. Harrison's Principles of Internal Medicine, 12th edn. New York: McGraw-Hill Inc, 1991:645-8.

13. Noordeen SK. Epidemiology and control of leprosy - A review of progress over the last 30 years. Trans R Soc Trop Med Hyg 1993;87:515-7.

14. Waters MFR. Chemotherapy of leprosy - Current status and future prospects. Trans R Soc Trop Med Hyg 1993;87:500-3.

15. Schuch D, Wolff L. Repair of mycotic aneurysm of the innominate artery with homograph tissue. Ann Thorac Surg 1991;52:863-4. 